Revue de l'Institut des langues et cultures

d'Europe, Amérique, Afrique, Asie et Australie

$32 \mid 2018$

Didactique des langues et cultures de spécialité :

méthodes, corpus et nouvelles technologies

\title{
El español para la negociación: aportaciones de la simulación al desarrollo de la competencia comunicativa de especialidad
}

L'espagnol pour la négociation: apports de la simulation au développement de la compétence communicative de spécialité

Spanish for Negotiation: Contributions of Simulation to the Development of Communicative Competence for Specific Purposes

\section{Sara Álvarez Martínez}

\section{OpenEdition}

Journals

Edición electrónica

URL: http://journals.openedition.org/ilcea/4753

DOI: $10.4000 /$ ilcea.4753

ISSN: 2101-0609

Editor

UGA Éditions/Université Grenoble Alpes

Edición impresa

ISBN: 978-2-37747-059-4

ISSN: $1639-6073$

\section{Referencia electrónica}

Sara Álvarez Martínez, «El español para la negociación: aportaciones de la simulación al desarrollo de la competencia comunicativa de especialidad », ILCEA [En línea], 32 | 2018, Publicado el 01 julio 2018, consultado el 01 mayo 2019. URL : http://journals.openedition.org/ilcea/4753 ; DOI : 10.4000/ ilcea. 4753

Este documento fue generado automáticamente el 1 mayo 2019.

(C) ILCEA 


\section{El español para la negociación: aportaciones de la simulación al desarrollo de la competencia comunicativa de especialidad}

L'espagnol pour la négociation: apports de la simulation au développement de la compétence communicative de spécialité

Spanish for Negotiation: Contributions of Simulation to the Development of Communicative Competence for Specific Purposes

Sara Álvarez Martínez

\section{Introducción}

1 La creciente proliferación de formaciones universitarias orientadas a la profesionalización en las últimas décadas ha llevado a reconsiderar las prácticas pedagógicas y los enfoques empleados en los másteres de lenguas extranjeras aplicadas (LEA) en Francia. Dado que uno de los objetivos principales de las materias lingüísticas que componen el cursus de dichas formaciones ${ }^{1}$ es acercar a los estudiantes a la realidad profesional de un ámbito específico, es tarea del discente revisar los métodos que mayor interés presentan a los estudiantes para llegar a ser hablantes competentes en la lengua y la cultura de especialidad metas. Tomando como punto de partida esta consideración, en el presente estudio nos centraremos en las técnicas que favorecen la práctica de la negociación en el marco del Máster en Negociación Trilingüe en Comercio Internacional (NTCI).

2 Sabido es que la negociación es una manifestación de la comunicación oral en la empresa de gran importancia para el desenvolvimiento de los negocios de cualquier medio empresarial. Por ello, consideramos que la ejercitación de la misma exige una caracterización previa de dicho proceso comunicativo que, a su vez, facilitará la 
integración de la realidad comunicativa de la negociación empesarial en el aula de español de los negocios (ENE) a través de recursos lingüísticos y no lingüísticos propios de las situaciones profesionales en las que se lleva a cabo el proceso negociador.

3 Los estudios que definen la negociación como proceso comunicativo (Malaret, 2003; Mercelot, 2006) ponen de relieve el hecho de que toda negociación comercial constituye una interacción -que surge en torno a un conflicto o a la existencia de divergenciasencaminada a la obtención de un acuerdo y, por tanto, a la búsqueda de soluciones satisfactorias para ambas partes. Saber negociar implica, por tanto, enfretarse a coacciones o acciones comunes con una finalidad colectiva empleando la lengua de especialidad adecuada en una situación concreta del ámbito profesional teniendo asimismo en cuenta los aspectos culturales del contexto en que se negocia. Galisson pone de relieve el hecho de que: «[...] le commun des mortels n'apprend pas une langue pour en démontrer les mécanismes et manipuler gratuitement des mots nouveaux, mais pour fonctionner dans la culture qui va avec la langue» (1998: 273).

La afirmación anterior adquiere una relevancia particular en el caso de estudiantes cuya aspiración es llegar a ser buenos negociadores en una lengua extranjera ya que su deseo es ser operativos en el mercado laboral y, por consiguiente, necesitan adquirir (además de conocimientos lingüísticos) el saber hacer propio al contexto profesional. Partiendo de esta reflexión, en el presente artículo trataremos de precisar en qué medida dos de las técnicas didáctivas activas aplicadas al aprendizaje del español facilitan el desarrollo de la competencia comunicativa de especialidad en el ámbito de la negogiación y, por ende, favorece la formación de buenos actores sociales capaces de construir sus propios saberes (Mercelot, 2006: 259). Para ello analizaremos un corpus de interacciones pedagógicas resultado de la puesta en práctica de dos tipos de simulación: el juego de rol y el estudio de casos.

\section{Referentes conceptuales}

El marco teórico de nuestro estudio hace referencia, por una parte, a las técnicas didácticas activas que nos han permitido obtener un corpus de interaccionesnegociaciones pedagógicas y, por otra parte, a la noción de competencia comunicativa de especialidad. Esta última es fundamental para determinar las aportaciones de dichos métodos a su desarrollo en el ámbito de la negociación.

\subsection{Las técnicas didáctivas activas en la clase de español de los negocios}

6 El afán por ofrecer formaciones orientadas a una profesionalización ha llevado a los docentes de Español para Fines Específicos (EFE) a explotar la llamada técnica heurística de la dramatización con el fin de que los aprendices empleen la lengua en situaciones profesionales meta. Estas situaciones deben estar inmersas en la realidad comunicativa del ámbito especializado que tratan de adquirir y dominar los alumnos y, por ello, se han adoptado métodos que toman los procedimientos de simulación como punto de partida (Gómez de Enterría, 2009: 106). La simulación aplicada al aprendizaje de lenguas extranjeras con fines profesionales ${ }^{2}$ alcanza gran auge en Francia en torno a los años 1970, donde posteriormente, en la década de los años 1980, se desarrollarán otras líneas de aplicación metodológica, basadas en simulaciones y enfocadas al aprendizaje de 
la lengua materna (Caré \& Debyser, 1995: 15). El objetivo de este tipo de métodos es resolver problemas reales que surgen en situaciones profesionales también reales, cuya finalidad es la resolución de casos mediante actividades y tareas predeterminadas. Es precisamente en estas situaciones comunicativas donde el alumno pone en práctica sus facultades lógicas co-cognitivas, gracias a las cuales ofrece una solución al problema planteado, al mismo tiempo que adquiere las competencias lingüística, comunicativa y cultural.

7 A través de la técnica de la dramatización se trata de buscar la manera de presentar, practicar, optimizar e impulsar el empleo de la lengua meta por parte de los alumnos, de tal manera que el aprendizaje de la lengua profesional sea lo más rentable y productivo para ellos y, por lo tanto, se estimule así tanto su motivación como su participación (Cabré \& Gómez de Enterría, 2006: 77).

8 Los métodos que favorecen la puesta en práctica de interacciones profesionales reales desarrollan procesos autónomos de pensamiento al mismo tiempo que facilitan la acumulación de experiencias lingüísticas propias del ámbito profesional elegido. Dichas experiencias solo pueden manifestarse en un contexto de interacción comunicativa en el que se pongan en práctica todas y cada una de las destrezas, habilidades, competencias y estrategias que son imprescindibles para lograr un nivel de comunicación óptimo, propuesto en el proceso de enseñanza-aprendizaje.

9 Tal y como hemos dicho en el apartado anterior, la negociación es un tipo de interacción cuyo desarrollo exige un soporte contextual de la realidad profesional, el cual favorece la práctica de los discursos especializados insertos en el funcionamiento de la comunicación. En este sentido, podemos realizar la siguiente hipótesis: los métodos que favorecen las dinámicas interactivas en las que se llevan a cabo procesos de negociación son aquellos que ponen en práctica técnicas didácticas activas centradas en la simulación como el juego de rol, el estudio de casos o la simulación global (SG)3.

De hecho, los procedimientos de simulación que interesan en el aula del ENE y, en particular, en las clases de negociación deben fundamentarse necesariamente en planteamientos metodológicos de carácter dinámico. En otras palabras, podemos decir que esta metodología activa va orientada hacia el funcionamiento de habilidades sociales (Cabré \& Gómez de Enterría, 2006: 90). La simulación en el ámbito de EFE traslada hasta el aula las realidades concretas que el futuro profesional debe conocer y analizar y, de esta manera, poder influir posteriormente sobre todo el proceso de aprendizaje. Así pues, la simulación resulta un ejercicio metodológico gracias al cual es posible llevar a cabo tareas simultáneas para enseñar todos los aspectos de la comunicación especializada.

11 En las próximas líneas nos detendremos en las dos dinámicas interactivas que han posibilitado la constitución de un corpus de interacciones-negociaciones, cuyos participantes fueron los estudiantes del Máster NTCI.

\subsubsection{Los juegos interactivos}

12 La teoría de los juegos, tradicionalmente empleada en el ámbito de las ciencias sociales para la resolución de problemas de diversos ámbitos económicos, se ha aplicado también en otros sectores (como el de la matemática, la filosofía, la biología, la ciencia política, etc.) con el fin de acercar los estudiantes a la resolución de conflictos. En realidad, los juegos de contratación o de negociación son una clara manifestación de este tipo de 
pedagogía que, a lo largo del siglo xx, cobrará cada vez más importancia en el ámbito de la enseñanza-aprendizaje de lenguas extranjeras.

En el ámbito de la enseñanza de lenguas extranjeras y, en particular, de lenguas para usos específicos son los juegos de rol aquellos que más se han explotado para lograr la interacción comunicativa en el aula, ya que en ellos se involucra a una grupo de participantes/alumnos con el objetivo de desarrollar habilidades específicas ${ }^{4}$. En este tipo de dinámicas el profesor ejerce un control sobre la actividad comunicativa desarrollada, lo cual va en detrimento de la creatividad del estudiante. Sin embargo, dicho control se efectúa únicamente sobre la situación y los papeles que representan los estudiantes, de modo que los participantes pueden expresarse libremente creando finalmente la interacción. Ahora bien, uno de los aspectos que mayor controversia ha generado respecto a los juegos de rol hace referencia a la falta de conexión con la realidad, al resultar, en algunos casos, faltos de contenido y suspendidos en un espacio intemporal ajenos a la realidad. Por ello, Littlewood (1996: 49) aboga por una programación de los juegos de rol con contenidos funcionales y lingüísticos, objetivos y procedimientos, que los acercan a ejercicios de simulación a pequeña escala. De esta manera, los juegos de rol facilitan la explotación en el aula de las cuatro destrezas lingüísticas, todas ellas tan necesarias para el buen desarrollo de las negociaciones en el contexto profesional.

\subsubsection{El estudio de casos centrados en la simulación ${ }^{5}$}

El estudio de casos presenta a los estudiantes una situación concreta, dando amplia información sobre las circunstancias que lo envuelven con el fin de analizarlo, resolverlo, elaborar conclusiones y proponer posibles vías de actuación para su resolución. Entre los distintos modelos de estudio de casos propuestos por Martínez y Musitu (1995: 5), es precisamente el modelo cuyo objetivo es el entrenamiento en la resolución de situaciones el que mayor interés suscita entre los profesionales de la enseñanza/aprendizaje de lenguas con fines profesionales. Dicho modelo consta a su vez de tres tipos de casos:

a. Los casos centrados en el estudio de descripciones, los cuales proponen que los estudiantes participantes se ejerciten en el análisis, la identificación y la descripción de los puntos clave constitutivos de una situación dada con el fin de debatir y reflexionar las distintas perspectivas de resolución.

b. Los casos de resolución de problemas, cuyo objetivo se centra en la toma de decisiones que requiere la solución de problemas planteados en la situación que se somete a revisión.

c. Los casos centrados en la simulación, en los cuales no solo se pretende que los participantes estudien el relato, analicen las variables que caracterizan el ambiente en que se desarrolla la situación, identifiquen los problemas y propongan soluciones, sino que además se busca que los participantes se sumerjan en la situación, se involucren y participen activamente en el desarrollo del caso y tomen parte en la dramatización de la situación representando el papel de los personajes que participan en el relato.

15 Es precisamente este último el que mayor interés presenta en las clases de negociación en una lengua extranjera. Mucchielli (1990) precisa las condiciones necesarias para que dicha perspectiva didáctica se lleve a cabo con éxito: la autenticidad de la situación planteada, la urgencia de la misma, la orientación pedagógica y la exhaustividad informativa.

16 Al igual que la SG, el estudio de casos centrado en la simulación debe plantearse a través de materiales y problemáticas del mundo real, lo cual hará posible la transferencia de los 
aprendizajes realizados en el proceso formativo a escenarios y situaciones de la vida profesional real. Esta metodología requiere una importante y precisa planificación y preparación por parte del profesor, lo cual comienza con la identificación de casos susceptibles de ser llevados al aula. Así pues, la calidad didáctica de los mismos dependerá, en primer lugar, de si son casos verosímiles, seductores y suficientemente provocadores para captar la atención y el interés de los estudiantes en su resolución. En segundo lugar, deben ser claros y precisos, proporcionando toda la información necesaria para su comprensión. En tercer lugar, conviene evitar un exceso de información que pueda anticipar posibles soluciones a la situación planteada. En cuarto lugar, se rechazarán aquellos casos que no presentan una visión completa de la situación, así como aquellos que dan pie a una interpretación subjetiva de la situación descrita. Y, por último, se darán prioridad a aquellos casos que incluyan preguntas críticas que orienten a una solución analítica centrada en el contenido del caso o a una solución reflexiva que vaya más allá de los límites de la situación propuesta.

\subsection{La noción de competencia comunicativa de especialidad}

17 La afirmación de Lhote (2001: 450) referente a la necesidad de enfocar el aprendizaje de una lengua extranjera en el hecho de saber interactuar con los demás adquiere una relevancia particular a la hora de enseñar el español para la negociación. Interacción, discurso y aprendizaje deben ir indisolublemente unidos en todo proceso de enseñanza de una lengua extranjera con fines profesionales (Moirand, 1982: 52) ya que el estudiante de EFE deberá conseguir un doble objetivo al final del curso: por una parte, un objetivo formativo o profesional y, por otra, un objetivo comunicativo. De esta manera, el discente tendrá que demostrar su competencia y su operatividad en el mundo del trabajo al que aspira de tal manera que pueda enfrentarse diariamente con una realidad profesional adquirida.

Teniendo en cuenta las necesidades anteriores, las formaciones orientadas a la profesionalización debieran ofrecer una formación profesional en lengua extranjera cuyo objetivo principal tendría que centrarse en el desarrollo de la competencia comunicativa (CC) tal y como la entiende Moirand (1990: 20). Esto es, una competencia compuesta por un componente lingüístico (conocimiento y apropiación de modelos fonéticos, léxicos, gramaticales y textuales), un componente discursivo (conocimiento y apropiación de los diferentes tipos de discurso y de su organización en función de los parámetros de la situación de comunicación en la que son producidos e interpretados), un componente referencial (conocimiento de los distintos ámbitos de experiencia y de los objetos del mundo) y un componente sociocultural (conocimiento y apropiación de las reglas sociales y de las normas de interacción entre los individuos y las instituciones, el conocimiento de la historia cultural y de las relaciones entre los objetos sociales).

Por su parte, Cabré y Gómez de Enterría (2006: 77) retoman esta idea al considerar que la CC en la lengua de especialidad debiera incluir la competencia lingüística (elementos semánticos), la competencia pragmática (funciones, actos de habla, conversación) y la competencia sociolingüística (convenciones socio-profesionales, intención comunicativa). Y añaden a dichos componentes la competencia estratégica, al considerar que el estudiante de EFE también se sirve de recursos verbales y no verbales con el objeto tanto de favorecer la efectividad en la comunicación como de compensar fallos que puedan 
producirse en ella, derivados de lagunas en el conocimiento que se tiene de la lengua o bien de otras condiciones que limitan la comunicación.

A este respecto Mercelot (2006: 270) considera que no se puede yuxtaponer los elementos lingüísticos y culturales ya que, según él, los modelos fonéticos, léxicos, textuales, discursivos y referenciales no existen de manera independiente a la socialización. Por ello, este autor insiste en que todos los componentes de la competencia comunicativa tienen una faceta cultural. Desde su punto de vista, la $\mathrm{CC}$ de especialidad está constituida por:

a. La competencia lingüística, formada no solo por los modelos fonéticos, léxicos, gramaticales y textuales del sistema de la lengua meta, sino también por el dominio de dichos modelos en las situaciones de producción.

b. La competencia comportamental, formada por el conocimiento y la comprensión de la proxemia (espacio, distancias, posturas, etc.), cinésica (gestos, mímica, mirada, etc.) y los artefactos de la lengua y la cultura metas ${ }^{6}$ así como el dominio de dichos aspectos en la situación de producción.

c. La competencia pragmática, que hace referencia al conocimiento y la comprensión de los diferentes tipos de discursos y de su organización, su sentido y sus efectos en la situación de comunicación en que aparecen;

d. La competencia referencial, que se refiere al conocimiento de los dominios de experiencia y de los objetos del mundo y de sus relaciones en la esfera donde se habla la lengua meta;

e. La competencia intercultural que funciona en interacción permanente con las cuatro competencias anteriores y que comprende las reglas sociales y las normas de interacción entre los individuos y las instituciones, así como su evolución y las relaciones entre los objetos sociales.

21 Desde nuestro punto de vista, en el aula de ENE y, en particular, de español para la negociación, debieran trabajarse cada una de las competencias anteriores con el fin de que los estudiantes sean capaces de interpretar en términos pragmáticos el comportamiento (lingüístico, no verbal, discursivo y cultural) de sus interlocutores y, por ende, ajustar su propia conducta a la de la cultura meta en el marco de la cual están negociando.

En el próximo apartado veremos en qué medida la técnica heurística de la dramatización permite que los estudiantes se familiaricen con los elementos de la CC de especialidad en el ámbito de la negociación.

\section{Metodología}

23 A continuación expondremos la constitución del corpus de interacciones que sustenta nuestro estudio, la justificación de la lingüística interaccional como metodología de análisis y las herramientas de las que nos hemos servido para el análisis en función del objetivo perseguido.

\subsection{Corpus}

24 Las interacciones-negociaciones pedagógicas que constituyen nuestro corpus son el resultado de simulaciones a partir de dos juegos de rol y dos estudios de caso centrados en la simulación. Tales técnicas fueron llevadas a cabo por 6 grupos de estudiantes ${ }^{7}$, lo cual dio lugar a 12 interacciones cuya duración oscila entre 40 y 60 minutos. La elección 
de estos dos tipos de técnica se explica fundamentalmente por la compatibilidad metodológica que ofrecen con la formación académica que los estudiantes están cursando en el momento en que participan a dichas negociaciones : el Máster Negociación Trilingüe en Comercio Internacional. El hecho de que se trate de una formación de máster en alternancia $^{8}$, cuyo calendario exige la presencia de los estudiantes-asalariados en la empresa donde realizan las prácticas cada 15 días, complica el poder llevar a cabo simulaciones más complejas, como es el caso de la SG, para cuyo desarrollo sería preciso dedicar la totalidad de horas lectivas ${ }^{9}$ de la materia en el marco de la cual se han desarrollado las negociaciones. Este contexto académico nos obliga a optar por técnicas que posibilitan la práctica de la negociación sin suponer una sobrecarga de trabajo para los participantes.

En cuanto a la tipología de las simulaciones, en la tabla 1 se recapitula la designación de cada negociación, el objetivo de la misma y los roles representados:

Tabla 1. - Recapitulación de simulaciones.

\begin{tabular}{|c|c|c|c|}
\hline Tipo de técnica & Designación & Objetivo de la negociación & Roles \\
\hline Estudio de caso & $\begin{array}{l}\text { Jamaica Coffee } \\
\text { Shop (JCS) }\end{array}$ & $\begin{array}{l}\text { La implantación de una franquicia } \\
\text { JCS en la ciudad de Grenoble }\end{array}$ & $\begin{array}{l}\text { - Franquiciadores de JCS } \\
\text { - Franquiciados } \\
\text { potenciales en: } \\
\text { a) el centro ciudad } \\
\text { (Place Grenette) } \\
\text { b) un centro comercial } \\
\text { (Grand Place) } \\
\text { c) en un lugar turístico } \\
\text { (La Bastille) }\end{array}$ \\
\hline Estudio de caso & Arteoliva & $\begin{array}{l}\text { La comercialización del aceite de } \\
\text { oliva español en envases } \\
\text { de plástico o vidrio en Francia e } \\
\text { Italia }\end{array}$ & $\begin{array}{l}\text { - Representantes de } \\
\text { Arteoliva } \\
\text { - Expertos en el envase } \\
\text { de plástico } \\
\text { - Expertos en el envase } \\
\text { de vidrio }\end{array}$ \\
\hline Juego de rol & $\begin{array}{l}\text { Jamones de } \\
\text { Aragón (JDA) }\end{array}$ & $\begin{array}{l}\text { Resolver el endeudamiento de JDA } \\
\text { en una situacion de suspensión de } \\
\text { pagos }\end{array}$ & $\begin{array}{l}\text { - Responsables de JDA } \\
\text { - Representantes de la } \\
\text { Banca Oscense de } \\
\text { Crédito } \\
\text { - Representantes de los } \\
\text { empleados } \\
\text { - Representantes de los } \\
\text { acreedores comunes }\end{array}$ \\
\hline
\end{tabular}




\begin{tabular}{|l|l|l|l|}
\hline Juego de rol & SAMCO-RAPID & $\begin{array}{l}\text { - Directivos del grupo } \\
\text { RAPID } \\
\text { individual brasileña por una } \\
\text { importante Corporación española } \\
\text { de material de construcción }\end{array}$ & $\begin{array}{l}\text { Político influyente y } \\
\text { cabeza del clan familiar } \\
\text { LAURI-SAMCO } \\
\text { - Abogado y asesor fiscal } \\
\text { de SAMCO } \\
\text { - Líder industrial de } \\
\text { LAURI }\end{array}$ \\
\hline
\end{tabular}

Todas las interacciones tuvieron lugar en el aula habitual de la asignatura «Lengua y cultura de especialidad» cuyo objetivo principal es trabajar los recursos lingüísticos y los aspectos culturales de la negociación en español. En todos los casos, la disposición del mobiliario de la clase se modificó para simular una negociación en una sala de reuniones en torno a una mesa redonda. Los estudiantes descubrieron el tipo de negociación dos semanas antes de su representación. En las interacciones resultado de los estudios de caso, los participantes tuvieron que familiarizarse con el supuesto planteado situándolo dentro del contexto específico en el que debía tener lugar, analizar las distintas perspectivas tratanto de señalar las principales variables a la situación planteada, detectar los puntos fuertes y débiles de la situación, estudiar separadamente cada uno de los problemas describiendo los principales cambios necesarios en cada situación, generar diversas alternativas de acción para abordar los cambios, estudiar los pros y los contras de cada alternativa, implementar la decisión tomada y proponer soluciones examinando los hechos y los acontecimientos narrados. En cuanto a los juegos de rol, la fase previa a la dramatización fue menos compleja ya que la labor de documentación de los estudiantes resultó menos laboriosa, al contar con un dossier donde se especificaban las instrucciones específicas para cada uno de los roles y la información adicional necesaria sobre el caso planteado Los juegos de rol hacían referencia a casos reales de negociación y su representación requería un estudio previo de los conflictos empresariales que habían llevado a las distintas partes negociadoras a afrontar situaciones extremamente complejas $^{10}$.

\subsection{Metodología de análisis}

Dado que nuestro objetivo es precisar las aportaciones de las técnicas del juego de rol y el estudio de casos al desarrollo de las competencias lingüística, comportamental, pragmática, referencial e intercultural en el ámbito de la negociación, hemos optado por adoptar un enfoque que nos brindara las herramientas necesarias para analizar tanto los elementos lingüísticos, interaccionales como no verbales e interculturales, significativos de las interacciones-negociaciones que componen nuestro corpus. Dicho enfoque hace referencia a la lingüística interaccional promulgada por Kerbrat-Orecchioni (1990, 1992, 1994 y 2004) y Vion (1992).

En la tabla 2 resumimos los elementos que han orientado nuesta investigación y que desarrollaremos en el apartado siguiente ${ }^{11}$ : 
Tabla 2. - Elementos para el análisis de las interacciones-negociaciones.

\begin{tabular}{|c|c|c|c|}
\hline \multicolumn{4}{|c|}{ PLANTILLA DE ANÁLISIS DE INTERACCIONES-NEGOCIACIONES PEDAGÓGICAS } \\
\hline $\begin{array}{l}\text { ELEMENTOS } \\
\text { LINGÜÍSTICOS }\end{array}$ & $\begin{array}{l}\text { ELEMENTOS } \\
\text { INTERACCIONALES }\end{array}$ & $\begin{array}{l}\text { ELEMENTOS } \\
\text { NO VERBALES }\end{array}$ & $\begin{array}{l}\text { ELEMENTOS } \\
\text { INTERCULTURALES }\end{array}$ \\
\hline $\begin{array}{l}\text { - Nivel morfosintáctico } \\
\text { - Nivel léxico }\end{array}$ & $\begin{array}{l}\text { - Actos de habla } \\
\text { - Gestión de turnos de } \\
\text { palabra } \\
\text { - Tipología de ecuencias } \\
\text { - Modos de transición } \\
\text { - Papeles } \\
\text { comunicativos } \\
\text { - Dimensión temática } \\
\text { - Modos de } \\
\text { organización } \\
\text { discurso } \\
\text { - La cortesía }\end{array}$ & $\begin{array}{l}\text { - Componente proxémico } \\
\text { - Comportamiento cinésico } \\
\text { - Artefactos } \\
\text { - Factores del entorno }\end{array}$ & $\begin{array}{l}\text { - Convenciones } \\
\text { - Normas de } \\
\text { comportamiento } \\
\text { - Reglas sociales }\end{array}$ \\
\hline
\end{tabular}

\section{Análisis de datos ${ }^{12}$}

En el presente apartado analizaremos las negociaciones pedagógicas con el fin de precisar las aportaciones de las técnicas de dramatización seleccionadas al desarrollo de la competencia comunicativa de especialidad en el ámbito de la negociación. El análisis cualitativo que presentamos se estructura en torno a cuatro niveles según la perspectiva de la lingüística interaccional: el nivel lingüístico, el nivel interaccional, el nivel no verbal y el nivel intercultural.

En lo que concierne al nivel lingüístico y, en particular, morfosintáctico cabe señalar el predominio de la $1^{\text {a }}$. persona del plural o bien de formas impersonales. Las acciones a las que se hacen referencia se expresan a través de verbos que podemos agrupar en las siguientes categorías: descripción de actividades profesionales (trabajar, realizar, redactar, evaluar), transacción comercial (firmar, transferir, cobrar), posesión (poseer, disponer de, tener), actividad intelectual (considerar, barajar, pensar, creer), deseo o intención (querer, desear, gustar), acuerdo o desacuerdo (ponerse de acuerdo, compartir, discrepar), presentación (exponer, explicar, abordar), expresión de la cantidad (añadir, dismunuir, restar ), expresión de la apariencia (parecer, tener la impresión, resultar) y la obligación (deber, tener que, convenir). Otro de los aspectos representativos a nivel morfológico es la importancia que tienen los adverbios en el discurso negociador sobre todo para indicar precisión (especialmente, particularmente), manera (así, de este modo), probalidad ( seguramente, probablemente, a lo mejor), tiempo (ya, luego, después, enseguida) o afirmación/ negación (sí, no, por supuesto, en ningún caso).

31 En cuanto a la sintaxis empleada, esta estará condicionada por el objetivo de la negociación. Así, por ejemplo, en las simulaciones JCS y Arteoliva, cuya problemática 
implica la selección de un lugar y un envase respectivamente, se aprecia el uso recurrente de la comparación, la obligación y el contraste, mientras que en JDA y SAMCO-RAPID, la finalidad es resolver situaciones empresariales conflictivas (endeudamiento y fusión, respectivamente) se opta por el uso de estructuras condicionales, la expresión de la hipótesis, la realización de propuestas y la voluntad de fijar condiciones. La sintaxis que caracteriza estas interacciones presenta la acumulación de enunciados que se formulan conforme vienen a la mente del que habla, enunciados que no son necesariamente independientes, dando pie a una sintaxis concatenada (1). Si bien los estudiantes han preparado previamente sus roles $\mathrm{y}$, por lo tanto, sus argumentaciones, el discurso se caracteriza por un continuo ir y venir intentando explicar sus ideas con detalle, de manera precisa, lo cual da lugar a rodeos explicativos que revelan un afán de parafrasear lo que uno mismo u otro interlocutor ya ha dicho. En este sentido, es importante señalar el papel metadiscursivo y metacomunicativo de los conectores pragmáticos, los cuales no solo sirven de apoyo a los interlocutores para formular y reformular sus mensajes, sino también para agarrar y ordenar las partes de los mismos. La abundancia de reformuladores metadiscursivos (2) de la propia argumentación (o sea, es decir, en otras palabras) es crucial para conectar intercambios no lineales y evitar así problemas de organización, de actuación y de comprensión. Las negociaciones pedagógicas analizadas ponen de relieve la importancia del uso de conectores con papel demarcativo (3), que ordenan las partes del discurso (en primer lugar, en segundo lugar... por último; por un parte, por otra parte, etc.), así como del uso de conectores metadiscursivos reformuladores, explicativos, correctivos, resumidores. La frecuencia de estos recursos lingüísticos nos llevan a calificar el discurso de las negociaciones pedagógicas como un discurso repetitivo, redundante y recapitulativo en el que los interlocutores recurren a la estrategia de la retroalimentación constantemente (4).

En cuanto al nivel léxico ${ }^{13}$, las simulaciones analizadas exigen a los estudiantesnegociadores que se familiaricen con los léxicos técnico y subtécnico en torno a los siguientes nudos conceptuales:

Tabla 3. - Nudos conceptuales de las interacciones-negociaciones pedagógicas.

\begin{tabular}{|l|l|l|l|l|l|}
\hline SIMULACIÓN & \multicolumn{4}{l}{ NUDOS CONCEPTUALES } \\
\hline JCS & $\begin{array}{l}\text { El sistema de } \\
\text { franquicias }\end{array}$ & $\begin{array}{l}\text { Los requisitos y las } \\
\text { particularidades de JCS }\end{array}$ & $\begin{array}{l}\text { La potencialidad de Grenoble para } \\
\text { una franquicia de venta y } \\
\text { degustación de café }\end{array}$ \\
\hline ARTEOLIVA & $\begin{array}{l}\text { Estrategia } \\
\text { expansión de de } \\
\text { Arteoliva }\end{array}$ & $\begin{array}{l}\text { El mercado del } \\
\text { envasado }\end{array}$ & $\begin{array}{l}\text { La comercialización del aceite } \\
\text { de oliva }\end{array}$ \\
\hline JDA & $\begin{array}{l}\text { La suspensión de } \\
\text { pagos }\end{array}$ & $\begin{array}{l}\text { La banca y el } \\
\text { endeudamiento }\end{array}$ & $\begin{array}{l}\text { Procedimientos judiciales } \\
\text { SAMCO-RAPID } \\
\text { La compra-venta } \\
\text { de una empresa }\end{array}$ & $\begin{array}{l}\text { La industria de la } \\
\text { construcción }\end{array}$ & $\begin{array}{l}\text { Problemas de crecimiento } \\
\text { empresarial }\end{array}$ \\
\hline
\end{tabular}


Los estudiantes-negociadores tienen la posibilidad de acceder a este tipo de léxico durante la fase de documentación y preparación de la negociación de modo que durante la interacción el lenguaje de especialidad fluye de manera natural. El uso del léxico específico contextualizado favorece la aparición de combinaciones restringidas (o colocaciones) en el discurso especializado (como por ej: coste de producción, vender al por mayor/menor, mercado meta, salud financiera, bienes de consumo, canon de entrada, suspensión de pagos, capital circulante, plazos de reembolso, montar un negocio), que no suelen presentar dificultades a los estudiantes. Sin embargo, la omnipresencia de los números, las cifras, los porcentajes, la lectura de gráficos, la referencia a operaciones matemáticas, resulta uno de los aspectos más espinosos para los estudiantes incluso a nivel de máster. Por otra parte, el registro empleado en algunos momentos presenta rasgos propios del español coloquial dando lugar al empleo de expresiones idiomáticas.

A continuación, nos detendremos en el análisis del nivel interaccional. En primer lugar, conviene precisar que los estudios de casos centrados en la simulación dan lugar a interacciones comunicativas mucho más dinámicas y acusadas que los juegos de rol. En realidad, si bien la asignación de un rol determinado y la obligación de respectar instrucciones precisas puede facilitar al estudiante-negociador el desarrollo de su argumentación, la otra cara de la moneda refleja que esta manera de proceder puede dar lugar a interacciones encorsetadas e, incluso, un tanto forzadas. En cambio, en el caso de las interacciones fruto de los estudios de casos centrados en la simulación, los estudiantes dan rienda suelta a sus argumentaciones y ello se refleja en un mayor grado de implicación en la negociación.

En cualquier caso, todas las interacciones-negociaciones muestran tres secuencias básicas:

- Inicio: secuencia compuesta por los saludos, las presentaciones, los motivos y/o los objetivos de la reunión y el orden del día (si lo hay);

- Desarrollo: secuencia en la que se exponen los intereses de cada una de las partes, las argumentaciones, las objeciones, los acuerdos y los desacuerdos, las concesiones, las propuestas, las condiciones..

- Cierre: secuencia en la que vemos las conclusiones del encuentro, las recapitulaciones de los puntos tratados, los agradecimientos, las despedidas.

Mientras que la primera y la tercera secuencias constituyen formas rituales de la negociación, la secuencia central es la que está sujeta a mayor flexibilidad y donde se produce la co-construcción del discurso. En ella se aprecian fundamentalmente dos modos de organización del discurso: la descripción y la argumentación. De ahí la importancia de los conectores pragmáticos como ordenadores del discurso (tal y como hemos señalado más arriba) pero también como portadores de instrucción argumentativa, ya sea para producir argumentos coorientados (si sirven a una misma conclusión) como antiorientados (si persiguen conclusiones inversas). En cuanto a las funciones argumentativas (5) más destacadas en nuestro corpus podemos destacar: la justificación, la oposición, la concesión y la conclusión.

Los roles representados no solamente influyen en el tipo de función argumentativa desarrollada, sino también en la diversidad de los actos de habla producidos. Así, por ejemplo, los roles relacionados con la dirección o las responsabilidades empresariales (como los franquiciadores en Arteoliva y JCS) dan pie a un predominio de actos directivos y asertivos (6) que conducen a los interlocutores que representan dichos roles a tomar las 
riendas de la interacción. Sin embargo, en el caso de que los directivos o responsables se encuentren en una situación poco favorable (como, por ejemplo, la suspensión de pagos en JDA), estos mismos roles propician la formulación de actos compromisorios y expresivos (7), cuyo objetivo es asumir compromisos con el fin de restaurar una relación comercial y mostrarse empáticos frente a los daños ocasionados. Este mismo tipo de actos de habla (asertivos y compromisorios) surgen con frecuencia en los discursos de los franquiciados potenciales en JCS y de los expertos en los envases de plástico y vidrio en Arteoliva. Ello muestra que la aserción y el compromiso son dos de las principales intenciones comunicativas de los negociadores que poseen una posición jerárquica inferior.

En cuanto a la gestión de los turnos de palabra y las alternancias, los estudiantesnegociadores se familiarizan con las distintas maneras de tomar el turno de palabra en función de su papel comunicativo pero también de su estilo negociador (dominante, conciliador, sumiso, crítico, inseguro). Así, por ejemplo, los participantes franquiciadores muestran una clara preferencia por la autoselección (8) mientras que los interlocutores franquiciados optan por la heteroselección (9). Del mismo modo, los estudiantes que representan roles directivos, en JCS y en JDA, son los que gestionan los turnos de palabra, interrumpen y marcan la dinámica interactiva de la negociación. En SAMCO, vemos cómo es la negociadora que representa el rol de político influyente y cabeza del clan familiar LAURI quien asume la labor de gestionar y distribuir los turnos de palabra sin que ello se haya pactado previamente. Por otra parte, conviene precisar que el dominio lingüístico y la fluidez también inciden en el origen de las tomas de palabra, lo cual se manifiesta en la preferencia de la autoselección por parte de aquellos estudiantes cuyo nivel de lengua es superior al de sus compañeros. En cualquier caso, la representación de ambas técnicas activas favorece la utilización de los distintos recursos para tomar el turno de palabra, para interrumpir y para finalizar su turno evitando el solapamiento. Asimismo, al igual que en una negociación real, los actores de las negociaciones pedagógicas se familiarizan con los diferentes modos de transición, esto es, deben hacer frente a pausas, a solapamientos o a interrupciones poniendo a prueba su habilidad comunicativa como en la vida real.

Los roles marcan también el tipo de contribuciones de los interlocutores. Así, por ejemplo, los franquiciadores / directivos / líderes formulan con mayor facilidad contribuciones directivas y de relanzamiento (10); mientras que los franquiciados / expertos en envases/ otros miembros de la empresa realizan preferentemente contribuciones reactivas (11). Conviene destacar que, en simulaciones donde dos o más estudiantes representan un mismo rol, son frecuentes las contribuciones continuativas (12) para completar información de una posición argumentativa a través de marcadores aditivos entre los miembros de una misma parte negociadora.

Para acabar con los aspectos interaccionales, quisiéramos detenernos en la importancia de las expresiones modalizadoras (13) en las interacciones-negociaciones analizadas. De hecho, en este tipo de discurso los atenuadores constituyen una constante por parte de todos los interlocutores, lo cual muestra la importancia de los diferentes procedimientos sustitutivos para reemplazar un elemento por otro que rebaja la fuerza del acto amenazador. De ahí que nos encontremos con un discurso plagado de desactualizadores (uso del condicional o del subjuntivo o del imperfecto para pedir algo), de procedimientos acompañantes (por favor, si no es demasiada molestia, se lo ruego), de enunciados preliminares para anunciar algo y preparar un acto que puede atentar contra la imagen ( 
¿puedo hacer una pregunta?), de reparaciones (a través de la excusa) y de minimizadores ( solo una cosita). El discurso negociador pedagógico es, sin lugar a dudas, un discurso cortés que presenta, sin embargo, algunas alternancias entre los tratamientos de tú y de usted produciéndose de este modo una fluctuación incoherente que contrasta con la persistencia de las expresiones modalizadoras desde el principio hasta el final de las interacciones.

41 Seguidamente abordaremos las cuestiones relativas al nivel no verbal $\mathrm{y}$, más precisamente, cuestiones que derivan de la proxemia y de la cinésica. En primer lugar, es importante señalar que ambos métodos (estudio de casos y juego de rol) posibilitan la transformación del aula en una sala de reunión. Así pues, la disposición de las mesas en círculo como si se tratara de una gran mesa redonda, el uso de ordenadores, de bloc de notas, de imágenes, de documentos para la negociación, la presencia de botellas de agua o de tazas de café, etc., todo ello lleva a los estudiantes a crear un contexto profesional muy cercano al que deben enfrentarse durante sus prácticas en régimen de alternancia. Es interesante precisar que, si bien todos los interlocutores están sentados en la misma mesa, la mayor o menor distancia que mantienen los participantes entre sí depende de su posición argumentativa. Esto es, los estudiantes-negociadores que comparten un mismo rol se sentarán más cerca los unos de los otros facilitándose así el susurro o la comunicación no verbal a través de la mirada con el fin de ayudarse, de aclarar algunos aspectos, de ponerse de acuerdo en algo, de gestionar un imprevisto, de solicitar la colaboración de sus colegas, etc.

En segundo lugar, este tipo de prácticas facilitan la toma de conciencia del comportamiento cinésico de los estudiantes-negociadores gracias al análisis posterior a la grabación de los gestos, las maneras y las posturas que ellos mismos adoptan durante la interacción. Es interesante analizar el significado de este tipo de lenguaje no verbal en la negociación. Veamos en la tabla 4 algunos de los elementos cinésicos y su significado en la negociación según el análisis realizado por los propios estudiantes:

Tabla 4. - Comportamiento cinésico en la negociación.

\begin{tabular}{|l|l|}
\hline \multicolumn{2}{|l|}{ ELEMENTOS CINÉSICOS EN LAS NEGOCIACIONES PEDAGÓGICAS } \\
\hline GESTO/MANERA/POSTURA & SIGNIFICADO \\
\hline Chocar la mano & Saludar antes y después de la negociación \\
\hline Movimiento circular de manos abiertas & $\begin{array}{l}\text { Completar lo que uno dice en momentos de falta de } \\
\text { fluidez }\end{array}$ \\
\hline Manos cruzadas inmóviles & Adoptar una postura firme \\
\hline Mostrar imágenes, gráficos, documentos & Ejemplificar lo que uno está explicando \\
\hline Mover la cabeza para arriba y para abajo & $\begin{array}{l}\text { Corroborar lo que dice otra persona, aprobar } \\
\text { una idea }\end{array}$ \\
\hline Mover la cabeza de izquierda a derecha & Mostrar desacuerdo \\
\hline
\end{tabular}




\begin{tabular}{|c|c|}
\hline $\begin{array}{l}\text { Buscar el contacto visual de los } \\
\text { interlocutores }\end{array}$ & $\begin{array}{l}\text { Asegurarse de que el canal de comunicación } \\
\text { funciona }\end{array}$ \\
\hline Enumerar con los dedos & Especificar distintos argumentos \\
\hline $\begin{array}{l}\text { Tener el bolígrafo entre los dedos y } \\
\text { moverlo continuamente }\end{array}$ & Mostrar nerviosismo \\
\hline $\begin{array}{l}\text { Mover las manos con la palma abierta } \\
\text { hacia arriba }\end{array}$ & Dar mayor énfasis a lo que estamos diciendo \\
\hline Sonreír mientras se habla & $\begin{array}{l}\text { Mostrar empatía para solucionar un malentendido } \\
\text { Atenuar una crítica, una petición, una objeción } \\
\text { Resolver un solapamiento }\end{array}$ \\
\hline $\begin{array}{l}\text { Codos apoyados en la mesa y manos } \\
\text { cruzadas en posición vertical }\end{array}$ & Reafirmar una posición argumentativa \\
\hline $\begin{array}{l}\text { Codos apoyados en la mesa y manos juntas } \\
\text { en posición vertical que se abren y se } \\
\text { cierran }\end{array}$ & Aclarar un malentendido \\
\hline $\begin{array}{l}\text { Dedos entrecruzados de forma horizontal } \\
\text { y movimiento de manos pausado }\end{array}$ & $\begin{array}{l}\text { Expresar calma, voluntad de acuerdo, posición } \\
\text { colaborativa }\end{array}$ \\
\hline Sonrojarse & Muestra de timidez o estrés al hablar en público \\
\hline Tocarse el mentón & $\begin{array}{l}\text { Adoptar una postura reflexiva en un momento } \\
\text { culminante de la negociación }\end{array}$ \\
\hline Ceño fruncido & Mostrar incomprensión, desacuerdo, estupefacción \\
\hline Hacer un chasquido & Indicar una laguna lingüística \\
\hline
\end{tabular}

En tercer y último lugar, quisiéramos incidir en la importancia de los artefactos en este tipo de interacciones a la hora de conferir mayor realidad a la negociación. A este respecto, conviene precisar que el día de la simulación los estudiantes-negociadores adoptan una vestimenta profesional (traje chaqueta, americana, camisa, vestido, zapatos de tacón) y se sirven de todo aquello que pudieran emplear en una sala de reuniones ( ordenador, calculadora, documentación, bloc de notas, dossieres, cafetera). De esta manera, el aula deja de ser un espacio académico y se convierte en un espacio profesional para la negociación.

Finalizaremos este apartado haciendo referencia al nivel intercultural. Ante todo, conviene precisar que las simulaciones realizadas no solamente son el telón de fondo para acceder a las convenciones y las normas de comportamiento típicas de una negociación (sobre todo al principio y al final de las interacciones: ritual de saludos, instalación del clima de confianza, empleo de fórmulas de cortesía, gestión de las distintas partes de la negociación...) sino que, además, gracias a ellas los estudiantes tienen la posibilidad de «descubrir» cuestiones culturales relacionadas con la problemática de la negociación 
durante la fase de documentación. Por ejemplo, el caso de JDA no solamente da pie a familiarizarse con las distintas vías que puede adoptar una empresa española en una situación de suspensión de pagos según el derecho concursal sino que, además, los estudiantes deben documentarse sobre la actividad de dicho empresa (la producción y comercialización de jamones ibéricos: pata negra, de bellota, 5 jotas, etc.). En Arteoliva, los estudiantes deben familiarizarse con los requisitos del envasado de un producto típico español (el aceite de oliva virgen extra), y con la competencia en otros mercados europeos como Francia e Italia. En JCS es importante conocer el sistema de franquicias en el ámbito de la restauración y, en particular, el sector de las cafeterías-torrefactoras en España. Por último, el interés de la negociación SAMCO-RAPID desde el punto de vista intercultural radica en el estudio previo que los estudiantes deben realizar de las culturas española y brasileña para llevar a cabo una compra-venta en el sector de la construcción.

\section{A modo de conclusión}

El análisis descriptivo cualitativo realizado nos acerca a la comprensión de que técnicas didácticas activas como el juego de rol y el estudio de casos pueden llegar a jugar un efecto favorable en el desarrollo de la competencia comunicativa en el ámbito de la negociación, a falta de estudios experimentales que confirmen su evidencia. Las interacciones-negociaciones recopiladas ponen de relieve el hecho de que ambos métodos favorecen el traslado al aula de realidades concretas del futuro profesional y ubican al estudiante-negociador en un contexto de aprendizaje activo que propicia el desarrollo de modos de organización como la argumentación, la contraargumentación, la reformulación y la retroalimentación, todos ellos necesarios y relevantes en cualquier proceso de negociación.

Además, el análisis revela que ambos métodos posibilitan la práctica de las cuatro destrezas necesarias en cualquier proceso negociador (interacción, comprensión oral, comprensión escrita, expresión escrita) al mismo tiempo que se facilita la exposición a los distintos aspectos de la comunicación especializada (lingüísticos, interaccionales, no verbales, interculturales) integrando y movilizando el saber-hacer necesario para enfrentarse a una negociación real.

Ahora bien, si comparamos las dinámicas interactivas en las que los hablantes representan roles determinados con aquellas en las que no se especifican los papeles, constatamos que la asunción de un rol puede, en algunos casos, encorsetar la interacción y limitar la participación de los estudiantes que no consigan conectar con la personalidad representada. Por esta razón, el poder asumir roles ya conocidos de empresas cercanas a los estudiantes podría resolver esta limitación. De hecho, cuando los estudiantes se identifican con sus personajes, estos logran experimentar diferentes personalidades, estados o características psicológicas, como por ejemplo, la amabilidad, la diplomacia, la impaciencia, la comprensión, la cortesía o el enfado; todas ellas relevantes y recurrentes a la hora de participar en una negociación.

Asimismo, dado que los estudiantes protagonistas de dichas negociaciones son también asalariados, sería interesante abordar en el aula problemáticas de las empresas donde desarrollan sus prácticas con el fin de lograr una mayor conexión con la realidad profesional. En este sentido, la existencia de estudios comparativos entre negociaciones empresariales reales y pedagógicas podría ayudarnos a completar las consideraciones extraídas en el presente estudio. 
En cualquier caso, ante la dificultad de poder constituir y analizar corpus de negociaciones comerciales reales que sirvan de modelo a futuros negociadores, el interés de analizar interacciones pedagógicas en torno a un proceso negociador reside en conocer los aspectos lingüísticos, interaccionales, no verbales y culturales que el profesor debiera trabajar previamente con los estudiantes-negociadores sin perder de vista las distintas funciones argumentativas, con el fin de que estos adquieran mayor confianza a la hora de enfrentarse a una negociación pedagógica y, posteriormente, real.

\section{BIBLIOGRAFÍA}

CABRÉ María Teresa \& GómEZ DE ENTERRIA Josefa (2006), La enseñanza de las lenguas de especialidad. La simulación global, Madrid: Gredos.

CABRÉ María Teresa (2009), «La Teoría Comunicativa de la Terminología, una aproximación lingüística a los términos», Revue française de linguistique appliquée, 14(2), 9-15.

Calsamiglia Blancafort Helena \& Tuson Valls Amparo (2004), Las cosas del decir. Manual de análisis del discurso, Barcelona: Ariel Lingüística.

CARÉ Jean-Marc \& DeBYSER Francis (1984), Simulations globales, Paris: CIEP/BELC, CréaCom.

CARÉ Jean-Marc \& DEBYSER Francis (1995), Simulations globales, Sèvres: Centre international d'études pédagogiques.

DEBYSER Francis (1974), «Simulation et réalité dans l'enseignement des langues», Le français dans le monde, 104-106.

DEBYSER Francis (1990), «Les simulations globales», Éducation et pédagogie, 10.

GALISSON Robert (1998), «Le "français langue étrangère" montera-t-il dans le train en marche de la "Didactique scolaire" ?», Études de linguistique appliquée, 11, 265-286.

García CARbonell Amparo \& WatTs Frances (1996), «Telematic Simulation and Language Learning», F. Wats \& A. García Carbonell (eds.): Simulation Now! Simulación ¡Ya! Learning through Experience: The Challenge of Change. El aprendizaje a través de la experiencia: el reto del cambio, Valencia: Diputació de València, 585-595.

GÓMEZ DE ENTERRIA SÁNCHEZ Josefa (2009), El español lengua de especialidad: enseñanza y aprendizaje, Madrid: Arco Libros.

Gómez de EnterRia Josefa, Ruiz Martínez Ana María \& Martin de Nicolas María del Mar (2008), La comunicación oral en la empresa, Madrid: Arco Libros.

Kerbrat-ORECCHIONi Catherine (1990), Les interactions verbales, t. 1, Paris: Armand Colin.

KeRBRAT-ORECCHIONi Catherine (1992), Les interactions verbales, t. 2, Paris: Armand Colin.

Kerbrat-OrecChioni Catherine (1994), Les interactions verbales, t. 3, Paris: Armand Colin.

KeRBRAT-ORECCHIONI Catherine (2005), Le discours en interaction, Paris: Armand Colin.

KNAPP Mark L. (1980), La comunicación no verbal. El cuerpo y el entorno, Barcelona: Paidós. 
Lноте Élisabeth (2001) «Pour une didactologie de l'oralité», Études de linguistique appliquée, 123-124, 445-453.

LITTLEWOOD William (1996), La enseñanza comunicativa de idiomas. Introducción al enfoque comunicativo, Cambridge: Cambridge University Press.

MALARET Juan (2003), Manual de negociación y Mediación. Negociaciones empresariales eficaces para juristas y directivos, Madrid: Colex.

MARTínez SÁnCHEZ Amparo \& Musitu OCHOA Gonzalo (1995), El estudio de casos para profesionales de la acción social, Madrid: Narcea S.A. de ediciones.

MERCELOT Gérard (2006), Négociations comerciales et objectifs spéficiques. De la description à

l'ensegnement des interactions orales professionnelles, Berlin: Peter Lang.

MERCELOT Gérard (2013), «Que peut apporter l'analyse d'interactions orales à l'enseignementapprentissage des langues sur objectifs spécifiques ?», Recherche et pratiques pédagogiques en langues de spécialité, 32(1), 130-141.

MOIRAND Sophie (1982), Enseigner à communiquer en langue étrangère, Paris: Hachette.

MoIRAND Sophie (1990), «Décrire des discours produits dans de situations professionnelles», J.-C. Beacco \& D. Lehmann (coords.), Publics spécifiques et communication spécialisée, Paris: Hachette.

Mucchielli Roger (1970), La dinámica de los grupos, Madrid: Ibérica Europea de Ediciones.

PÉREZ GUTIÉRREZ Manuel (2001), «La simulación como técnica heurística en la clase de español con fines profesionales», Español para Fines Específicos. Actas del I CIEFE, Amsterdam: Consejería de educación y ciencia en Bélgica, Países Bajos y Luxemburgo, 169-183.

Vion Robert (1992), La communication verbale, Paris: Hachette.

WARSSERMANN Selma (1994), El estudio de casos como método de enseñanza, Buenos Aires: Amorrortu. YAICHE Francis (1996), Les Simulations globales : mode d'emploi, Paris: Hachette FLE.

\section{ANEXOS}

(1) Sintaxis concatenada

Solo me gustaría añadir que no se rompen las botellas de plástico y eso es una ventaja pienso en comparación con el vidrio que si una botella cae, puede romperse y para el transporte es una gran ventaja. (Arteoliva)

Lo siento, quiero saber ... Bueno, me parece que en general, en todos los casos en Francia, no se encuentran muchos envases de plástico para las aceitunas y para las salsas entonces ¿quéles parece como se podría solucionar esto? (Arteoliva)

(2) Reformuladores metadiscursivos

Es decir, que si la caída de la botella ocurre después de la compra el cliente aunque no tiene más la botella perfecta todavía tiene el producto que era el motivo principal de la compra. (Arteoliva)

Si me permiten resumir, tendríamos una participación del banco, de la Caja Oscense, perdón, de 2\%, nosotros estamos dispuestos, si los empleados nos apoyan, a aumentar esta cantidad, aumentar una participación del 5\%, si ustedes redujeran más la deuda, no solo los intereses ... un parte de la 
deuda de un 5\% sabiendo que una parte de la empresa tiene muy buenas perspectivas para los próximos años. (JDA)

(3) Conectores con papel demarcativo

Antes de continuar con la explicación del proyecto de cada uno, quisiéramos recordarles el perfil requerido de nuestros franquiciados [...] Así pues, para empezar, esperamos cierta capacidad financiera, en el mismo sentido, además se espera experiencia profesional en el comercio y la gestión de tiendas y, para acabar, se requiere que se identifique con nuestro comercio. (JCS)

Al orden del día hoy tenemos seis puntos: primero, la determinación del precio y el valor de la empresa; segundo, la participación accionarial entre el grupo RAPID y la familia Lauri; tercero, los problemas de crecimiento; cuarto, la presidencia del consejo; quinto la incorporación o no de la compra de división de tejas sexto y último, el tipo de sociedad que vamos a crear con la compraventa del grupo SAMCO; [...] (SAMCO-RAPID)

(4) Retroalimentación

- Para mí ... A mí me gustaría ceder solamente 49\% de la empresa para que pueda continuar teniendo un control sobre las decisiones ya que tenemos una imagen de empresa familiar ante nuestros clientes y es muy importante seguir con la política de la empresa así que me gustaría conservar la mayoría de la empresa.

- Si entiendo bien, conservaría el 51\% de la empresa. (SAMCO-RAPID)

(5) Funciones argumentativas

- Nos parecería legítimo que, que... dado los esfuerzos que han hecho los trabajadores socios, nos parecería legítimo que merezcan ser recompensados por esos esfuerzos, quizás una solución puede ser dar más participación en el capital o un cierto porcentaje sobre los beneficios.

- Pienso que, como lo hemos explicado antes, tenemos un porcentaje del 15\% con respecto respecto los beneficios, no tenemos miedo, si obtenemos beneficios, tendremos que pagar nuestras deudas, como los proveedores, que son indispensables, eh además, eh nosotros queremos ... obviamente no tenemos el dinero suficiente para la plantilla, os debemos cierta candidad de dinero y nos vemos obligados a reducir la plantilla de 5 trabajadores [pausa, silencio] entonces, el pedirnos ahorita un aumento sabiendo que no podemos..

- [...] Lo que quería decir mi colega es que los trabajadores que nunca han estado en conflicto con vosotros y que han pagado el régimen general de la seguridad social aunque a la empresa no... aunque no les pagan hace más de 10 meses, pensamos que están... son un montón de cosas ilegales... y si no es posible obtener una una una parte importante en las cuotas de la empresa, tal vez podemos obtener una garantía de la seguridad de los empleos pero no se despide a 5 empleados.

- En este caso hay que ver con vosotros si aceptáis la proposición de los colegas del banco, es que ya no tenemos dinero y esta solución puede ser una solución para recaudar un poco de... de... de... fondos y, en este caso, podría ayurdarnos a aseguraros la seguridad de los empleos. (JDA)

(6) Actos directivos y asertivos

[...] Pues como saben nuestro desarrollo se basa en la apertura de franquicias desde 1994 y somos la cadena líder del mercado español en el sector de las cafeterías especializadas... En la actualidad 
queremos abrir una franquicia en la ciudad de Grenoble y, por eso, estamos aquí para escuchar sus argumentos y elegir la propuesta más adecuada en relación con lo que buscamos [...] Así pues cuando quieran pueden empezar. (JCS)

\section{(7) Actos compromisorios}

Nos comprometemos a pagarles la deuda en un plazo máximo de 10 años. Otra posible solución podría ser el pago del 20\% de la deuda en este momento, que es mucho más de lo que ustedes nos han pedido, que nos han pedido el 15\%, y después durante 10 años podemos tener ciertas cuotas, también proponemos de acuerdo a los beneficios que vayamos a tener en los próximos años, podemos aumentar este procentaje. (JDA)

(8) Autoselección

Si me permiten añadir, como abogado y asesor de la familia, estoy aquí para representar a mis clientes $y$ ayudarles en sus decisiones y en cuanto al precio y valor de la empresa, pienso que es realmente clave no sobreevaluar el precio de la empresa, no se puede utilizar el valor contable de la empresa sino la imagen que ya tiene en Brasil [...] En cuanto a la presidencia del consejo, es importante tener un sitio en este consejo para mantener la imagen de una empresa brasileña pero con una inversión extranjera. (SAMCO-RAPID)

Disculpa yo tengo una pregunta con respecto a su producto, con respecto a la temperatura, como ya se sabe los productos no pueden ser alterados pero el envase, sobre todo el envase de plástico, no acepta mucho el cambio de temperatura, es decir, a más de una temperatura máxima los componentes del plástico comienzan a derritirse, entonces yo quería saber el nivel del transporte en verano: ¿Cómo hacen? ¿Cómo es el transporte? ¿Cómo mantienen el producto? (Arteoliva)

(9) Heteroselección

Pues propongo que cada uno exponga sus ideas para empezar, podría estar bien ver lo que cada uno quiere de una manera general y después podremos ver los puntos comunes. (SAMCO-RAPID)

Vamos ahora a escuchar cuáles son sus propuestas, ¿quién quiere empezar? (JCS)

(10) Contribuciones directivas y de relanzamiento

Me gustaría recuperar el punto sobre el valor de este bien, gracias por aportar esta solución... para aportar esta solución, me parece que si hoy aportamos 180 millones de euros, no estamos seguros de que dentro de 3 o 5 años ustedes tendrán el mismo valor... eso quiere decir que podemos perder dinero si sus bienes pierden valor entonces como el precio del mercado va a cambiar nos gustaría fijar otro valor, entonces proponemos que la suma de la hipoteca sea más bien de ... 100 millones de euros en vez de 180 millones... (JDA)

Yo quiero recordaros nuevamente que hemos decidido acogernos a la ley de suspensión de pagos, esto quiere decir que, si el gobierno, si el juzgado nos acuerda esto, nosotros no tendríamos ninguna oligación de pagarles la deuda. Sin embargo, nosotros queremos permanecer... tener una buena relación con ustedes. La Caja Oscencse nos ha ayudado en momentos de crisis ... eh ... no tenemos ninguna solución a parte una seguna hipoteca sobre los 800 000. (JDA)

(11) Contribución reactiva 
- Por ejemplo, tengo solo un ejemplo del agua San Pelegrino que es una agua italiana y que fue envasada en botella de plástico.

- Sí, pero es agua.

- Sí, ya sé que es agua, pero...

- Pero estamos hablando del aceite de oliva.

- No, porque la imagen es buena, la imagen de plástico y...

- Para el agua, para el agua... (Arteoliva)

(12) Contribución continuativa

- Solo me gustaría añadir que no se rompen las botellas de plástico y eso es una ventaja, pienso, en comparación con el vidrio, que si una botella cae, puede romperse y para el transporte es una gran ventaja.

-Y, por lo tanto, no se pierde el producto ... en las botellas de vidrio se pierde el producto pero en las botellas de plástico, claro que las botellas pueden ser dañadas pero van a permitir perder el producto en el interior. (Arteoliva)

(13) Expresiones modalizadoras

[...] Nos gustaría fijar otro valor, entonces proponemos que la suma de la hipoteca sea más bien de ... 100 millones de euros en vez de 180 millones... (JDA)

- Me gustaría añadir algo, bueno, pienso que usted debería quizás revisar, mmmmmm, volver a revisar su política de pago con sus clientes otorgándoles un plazo de pago más largo para tener dinero lo antes posible, es un punto a abordar.

- Quieres... quieres decir más corto.

- Sí, más corto.

- Porque acaba de decir un plazo de pago más largo. [sonrisas] (JDA)

- Muchas gracias por esta precisión, quizás si no le molesta, vamos a ver lo que nos pueden proponer ... los del plástico.

- Y, por favor, nos gustaría saber un poco más en cuanto a la conservación. (Arteoliva)

\section{NOTAS}

1. Las materias lingüísticas a las que hacemos alusión son: lengua y cultura de especialidad, estudio de casos y comunicación profesional.

2. Para más información sobre la simulación como método de formación y aprendizaje véase Cabré y Gómez de Enterría (2006: 83-88).

3. Si bien la simulación global es considerada el modelo de simulación más adecuado para el aprendizaje del español de especialidad (Gómez de Enterría, 2009:122; Cabré \& Gómez de Enterría, 2006: 80-89; Caré \& Debyser, 1995:15), su aplicación requiere una flexibilidad institucional que, en nuestro caso, no ha sido factible. Por ello, más adelante nos centraremos en los otros dos métodos que hemos podido poner en práctica en el marco del máster NTCI. 
4. Además de los juegos de rol, existen otros tipos de juegos interactivos insertos en la simulación: los juegos encaminados al desarrollo de conductas prácticas y los juegos que simulan situaciones reales (Cabré \& Gómez de Enterría, 2003: 93). Sería interesante experimentar estas otras dinámicas en el aula de ENE y comparar sus ventajas e inconvenientes en el ámbito de la negociación con respecto a los juegos de rol.

5. Como veremos más adelante, este tipo de estudio de casos se diferencia de aquel centrado en el estudio de descripciones y de los casos de resolución de problemas, en los que no hay simulación. 6. Siguiendo la nomenclatura de Knapp (1980), Calsamiglia Blancafort y Tusón Valls (2004), entendemos por artefactos uno de los factores no verbales de interés para el estudio de la comunicación humana, que hace referencia a aspectos como el perfume, la ropa, las pinturas, las gafas, las pelucas y los adornos (collares, anillos, sortijas, etc.).

7. El nivel de español de los estudiantes era heterogéneo ya que en algunos grupos participaron hablantes nativos. Así pues, podemos decir que el nivel de lengua oscilaba entre B1.2 y C1.

8. El régimen de alternancia implica que los estudiantes sean asalariados $\mathrm{y}$, por tanto, compaginen su formación académica con un período de prácticas cuyo calendario requiere su presencia en la empresa cada dos semanas.

9. Se trata de 18 horas lectivas por semestre, distribuidas en clases de 3 horas cada 15 días durante 9 semanas.

10. Los juegos de rol propuestos (Jamones de Aragón y SAMCO-RAPID) pueden consultarse en el Manual de negociación y mediación de Juan Malaret.

11. Respecto a los elementos lingüísticos, en este artículo nos centraremos en el análisis de la morfosintaxis y el léxico. Al tratarse de una situación de comunicación exolingüe consideramos que el análisis de los aspectos fonéticos y prosódicos no es pertinente aquí.

12. En el anexo el lector encontrará los extractos que ejemplifican los diferentes puntos del análisis. Dichos ejemplos aparecen numerados en el cuerpo del texto.

13. No es nuestro objetivo realizar un análisis exhaustivo del léxico empleado ya que ello requeriría un estudio independiente. Nos contentaremos con presentar los aspectos léxicos más representativos del discurso negociador propiciado por los métodos empleados.

\section{RESÚMENES}

El marco del presente artículo hace referencia a la didáctica de la comunicación profesional oral y, en particular, de la negociación en español. Tomando como punto de partida el análisis de un corpus de interacciones pedagógicas, resultado de dos tipos de técnicas de dramatización (el juego de rol y el estudio de casos) basadas en la simulación, nuestro objetivo es contribuir a precisar las aportaciones de dichos métodos al desarrollo de la competencia comunicativa de especialidad en el ámbito específico de la negociación empresarial. Para ello, analizaremos las interacciones-negociaciones pedagógicas sirviéndonos de las herramientas que proporciona la lingüística interaccional.

Dans le cadre de la didactique de la communication professionnelle orale et, en particulier, de la négociation en espagnol, notre objectif est de contribuer à préciser les apports au développement de la compétence communicative de spécialité dans le domaine de la négociation commerciale de deux techniques de dramatisation basées sur la simulation. Nous analyserons un corpus 
d'interactions pédagogiques, résultat de la mise en scène de deux jeux de rôle et deux études de cas orientés à la simulation, en nous servant des outils de la linguistique interactionnelle.

The present work lies within the scope of the didactics of teaching oral communication for professional purposes, in particular, negotiation skills in Spanish. Its aim is to examine the contribution of two drama technics implying simulation to the development of communicative competence in Spanish for trade negotiations. Our corpus of analysis is based on pedagogical interactions comprising two role plays and two simulation case studies, which serve as interactional linguistics tools.

ÍNDICE

Mots-clés: espagnol pour la négociation, simulation, jeu de rôle, étude de cas, compétence de spécialité, linguistique interactionnelle

Palabras claves: español para la negociación, simulación, juego de rol, estudio de caso, competencia de especialidad, lingüística interaccional

Keywords: Spanish for negotiation, simulation, role play, case study, language competence for professional purposes, interactional linguistics

\section{AUTOR}

SARA ÁLVAREZ MARTÍNEZ

ILCEA4, Université Grenoble Alpes 